\title{
Behaviour of organised disturbances in fully developed turbulent channel flow
}

\author{
P K SEN and SRINIVAS V VEERAVALLI
}

Department of Applied Mechanics, Indian Institute of Technology, New Delhi 110016 , India

e-mail: \{pksen,svrvalli\}@am.iitd.ernet.in

MS received 16 July 1999; revised 5 June 2000

\begin{abstract}
In our earlier work we have shown the relevance of stability theory in understanding the sustenance of turbulence in turbulent boundary layers. Here we adopt the same model to study the evolution of organised disturbances in turbulent channel flow. Since the dominant modes are wall modes we find that the stability characteristics in the two flows are nearly identical although the boundary conditions (at the edge of the boundary layer and at the centre of the channel) are different. Comparisons with the experiments of Hussain and Reynolds are also presented.
\end{abstract}

Keywords. Organised disturbances; turbulent channel flow; hydrodynamic stability; non-isotropic eddy viscosity model.

\section{Introduction}

In Sen \& Veeravalli (1998) (hereinafter referred to as S \& V), we examined the relevance of hydrodynamic stability theory to understanding fully developed turbulence in wallbounded flows. While the role of hydrodynamic stability theory in predicting the dominant instability mechanism in free shear flows is well-known and well-studied (see for example Gaster et al 1985, Liu 1988 and Roshko 1992), its usefulness in wall-bounded flows has remained in question.

Malkus (1956) suggested that if the mean velocity profile found in fully developed turbulent wall flows were used in the classical Orr-Sommerfeld equation then it should be marginally or neutrally stable at the flow Reynolds number. Reynolds \& Tiederman (1967) examined this conjecture and showed quite conclusively that it was not valid. Reynolds \& Hussain (1972) continued the work of Reynolds \& Tiederman (1967) using an isotropic eddy viscosity model in place of molecular viscosity. They derived an augmented OrrSommerfeld equation using what they call a Newtonian eddy model, with extra terms dependent on eddy viscosity and its spatial derivatives. However, their calculations again yielded damped modes.

$\mathrm{S} \& \mathrm{~V}$ continued along similar lines but used a more realistic eddy viscosity model, capable of yielding a non-isotropic Reynolds-stress tensor, based on the work of 
Pope (1975). With this model, S \& V obtained an extended Orr-Sommerfeld equation which exhibits unstable modes over a wide range of spatial wave numbers for the fully developed turbulent boundary layer at zero pressure gradient. Some of the key features of turbulent boundary layers like the location of the production peak are captured by this model.

Here we wish to study the evolution of organised disturbances in turbulent channel flow using the model presented in S \& V. Although the extended Orr-Sommerfeld equation is the same as that used in the boundary layer flow in $\mathrm{S} \& \mathrm{~V}$, the boundary conditions at the centre of the channel are different. However, since the unstable modes obtained in $\mathrm{S} \& \mathrm{~V}$ are wall modes, it would seem likely that the stability behaviour would be nearly the same. As we shall see below, it is indeed found to be the case. Some comparisons with the experimental results of Hussain \& Reynolds (1972) are also presented.

\section{Theory}

In the discussion to follow the instantaneous velocity vector is $u_{i}$ and the pressure is $p$. All physical quantites have been normalised by the average velocity, $V$, the channel half width, $H$, the kinematic viscosity, $\nu$, and density, $\rho$. The characteristic Reynolds number $\operatorname{Re}$ is defined as $V H / \nu$. The mean velocity is in the $x$ direction, $y$ is normal to the wall, and the mean flow is independent of the $z$ direction. $\left(x_{1}, x_{2}, x_{3}\right)$ and $(x, y, z)$ will be used interchangably and so also $\left(u_{1}, u_{2}, u_{3}\right)$ and $(u, v, w)$. Figure 1 shows the definition sketch of the flow.

We use the standard Reynolds decomposition:

$$
u_{i}=\bar{u}_{i}+u_{i}^{\prime} ; \quad p=\bar{p}+p^{\prime}
$$

where, $\bar{u}_{i}, \bar{p}$ are respectively the time averaged velocity and pressure, and $u_{i}^{\prime}, p^{\prime}$ are the respective turbulent fluctuations.

We now superimpose an organised (solenoidal) disturbance, $\tilde{u}_{i}, \tilde{p}$ (with zero mean), the instantaneous velocity and pressure then are respectively:

$$
u_{i}=\bar{u}_{i}+\tilde{u}_{i}+u_{i}^{\prime} ; \quad p=\bar{p}+\tilde{p}+p^{\prime} .
$$

The time averages of $u_{i}$ and $p$ remain the same but the ensemble (phase locked) averages are different. We denote ensemble averages by angular brackets \langle\rangle . Then,

$$
\left\langle u_{i}\right\rangle=\bar{u}_{i}+\tilde{u}_{i} ; \quad\langle p\rangle=\bar{p}+\tilde{p} .
$$

We assume that the organised disturbance is small in the following sense,

$$
\left|\left\langle\tilde{u}_{i} \tilde{u}_{j}\right\rangle\right|<<\left|\left\langle u_{i}^{\prime} u_{j}^{\prime}\right\rangle\right| \text {. }
$$

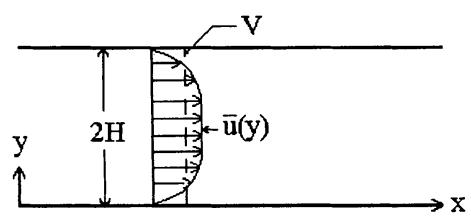

Figure 1. Definition sketch of the channel flow. 
We now introduce (twice) the rate of strain rate tensor and (twice) the vorticity tensor, respectively $s_{i j}$ and $\omega_{i j}$, as follows:

$$
s_{i j}=\left(\frac{\partial u_{i}}{\partial x_{j}}+\frac{\partial u_{j}}{\partial x_{i}}\right) ; \quad \omega_{i j}=\left(\frac{\partial u_{i}}{\partial x_{j}}-\frac{\partial u_{j}}{\partial x_{i}}\right) .
$$

The mean and ensemble averages are $\bar{s}_{i j}, \bar{\omega}_{i j}$ and $\left\langle s_{i j}\right\rangle$ and $\left\langle\omega_{i j}\right\rangle$ respectively.

As in S \& V, following Pope (1975), we model the time-averaged Reynolds stress tensor as follows:

$$
-\overline{u_{i}^{\prime} u_{j}^{\prime}}=-\frac{2}{3} k \delta_{i j}+\epsilon \bar{s}_{i j}-\epsilon C\left(k / \epsilon_{d}\right)\left[\frac{1}{2}\left[\bar{\omega}_{i k} \bar{s}_{k j}-\bar{s}_{i k} \bar{\omega}_{k j}\right]\right]
$$

Here, $\epsilon$ is the eddy viscosity defined as $-\overline{u^{\prime} v^{\prime}} /(\mathrm{d} \bar{u} / \mathrm{d} y) ; k$ is the turbulent kinetic energy; $\epsilon_{d}$ is the dissipation rate of the turbulent kinetic energy and $C$ is a constant. From the form of the above equation we can define an anisotropy parameter $\lambda$ as $C\left(k / \epsilon_{d}\right)$ $\mathrm{d} \bar{u} / \mathrm{d} y$. Thus the standard isotropic form of the eddy viscosity model is recovered when $\lambda$ is zero.

The ensemble average of the Reynolds stress similarly is modelled as:

$$
-\left\langle u_{i}^{\prime} u_{j}^{\prime}\right\rangle=-\frac{2}{3} k \delta_{i j}+\epsilon\left\langle s_{i j}\right\rangle-\epsilon\left(\lambda /(\bar{u})^{\prime}\right)\left[\frac{1}{2}\left[\left\langle\omega_{i k}\right\rangle\left\langle s_{k j}\right\rangle-\left\langle s_{i k}\right\rangle\left\langle\omega_{k j}\right\rangle\right]\right] .
$$

Here, $\bar{u}^{\prime}$, is the mean velocity gradient.

The instantaneous velocity $u_{i}$ obeys the Navier-Stokes and continuity equations for incompressible flow and from this the evolution equations for $\left\langle u_{i}\right\rangle$ may be obtained by ensemble averaging, and those for $\bar{u}_{i}$ by time averaging. If the equations for $\bar{u}_{i}$ are subtracted from the corresponding equations for $\left\langle u_{i}\right\rangle$, we obtain the required evolution equations for $\tilde{u}_{i}$ as:

$$
\frac{\partial \tilde{u}_{i}}{\partial t}+\bar{u}_{j} \frac{\partial \tilde{u}_{i}}{\partial x_{j}}+\tilde{u}_{j} \frac{\partial \bar{u}_{i}}{\partial x_{j}}=-\frac{1}{\rho} \frac{\partial \tilde{p}}{\partial x_{i}}+\nu \frac{\partial^{2} \tilde{u}_{i}}{\partial x_{j} \partial x_{j}}+\frac{\partial \tilde{r}_{i j}}{\partial x_{j}},
$$

and

$$
\frac{\partial \tilde{u}_{i}}{\partial x_{i}}=0
$$

where

$$
\tilde{r}_{i j} \equiv-\left(\left\langle u_{i}^{\prime} u_{j}^{\prime}\right\rangle-\overline{u_{i}^{\prime} u_{j}^{\prime}}\right)
$$

The model for $\tilde{r}_{i j}$ is obtained by subtracting (6) from (7).

Now, in order to obtain the general solution to (8), we need to consider normal modes of the form,

$$
\tilde{u}_{i}=\hat{u}_{i}(y) \exp ^{[\iota(\alpha x+\beta z-c \alpha t)]} .
$$

Here, $\hat{u}_{i}$ is the eigenfunction, $\alpha$ and $\beta$ are spatial wave numbers in the $x$ and $z$ directions respectively, and $c=c_{r}+\iota c_{i}$ is the complex wave speed.

The approach usually adopted is to take recourse to Squire's theorem and consider only 2-D disturbances i.e. with $\beta=\hat{w}=0$. If Squire's transformation, given by,

$$
\alpha^{*}=\left(\alpha^{2}+\beta^{2}\right)^{1 / 2} ; \quad \alpha^{*} u^{*}=\alpha \hat{u}+\beta \hat{w} ; \quad \alpha^{*} \operatorname{Re}^{*}=\alpha \operatorname{Re} \text { etc. }
$$




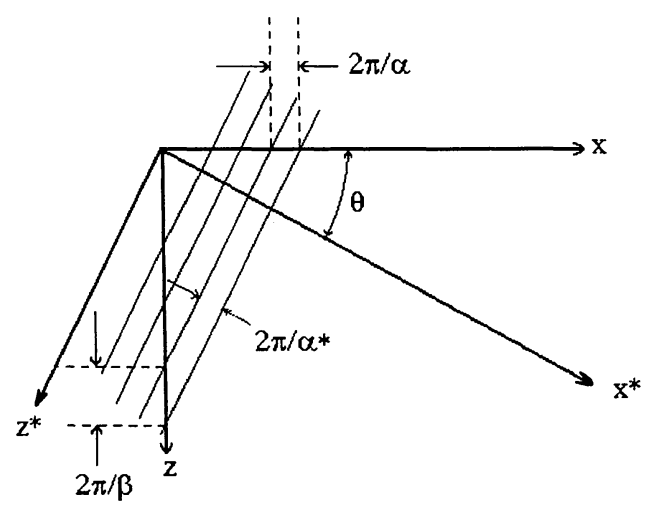

Figure 2. Oblique wave $(\alpha, \beta)$ with wavelength $\alpha^{*}$ in the oblique direction at angle $\theta$ with respect to the $\mathrm{x}$ axis.

is used in the present equations then one finds that the transformed equations for 3-D disturbances are not the same (in form) as those for 2-D disturbances. Thus Squire's theorem is strictly not valid. The difference arises only in the terms containing $\lambda$. However, if $\beta / \alpha \ll 1$ then it can be shown that the extra terms in the Squire-transformed 3-D equations are of higher order as compared to those corresponding to the 2-D problem. Another way to view this is to consider slightly oblique waves, making a small angle $\theta$ with the $x$ axis. Then (see figure 2),

$$
\alpha=\alpha^{*} \cos \theta \approx \alpha^{*} ; \quad \beta=\alpha^{*} \sin \theta \approx \theta \alpha^{*} \text { etc. }
$$

It can then be shown that the problematic terms in the Squire-transformed equations for the 3 -D case are of order $\theta^{2}$ - details may be found in appendix A. Thus, we can conclude that if we restrict $\beta$ to a small neighbourhood around zero then the most unstable modes correspond to $\beta=0$. Further, since we are interested in comparisons with experiments, wherein a 2-D disturbance has been used, we will restrict ourselves to 2-D disturbances in this paper. We note, however, that with the anisotropic model (as in (6) and (7)), for a complete solution to (8), the 3-D problem cannot be escaped.

We anticipate that the most likely 3-D structures are the resonant-triad (C-type) structures of Craik (1971), where two oblique waves of type $\left(\frac{1}{2} \alpha, \beta\right)$ and $\left(\frac{1}{2} \alpha,-\beta\right)$ are chosen whose $c_{r}$ value is the same as that of the corresponding 2-D wave of type $(\alpha, 0)$. The reason being that the nonlinear interaction of the two oblique waves produces a wave of type $(\alpha, 0)$ which refurbishes the 2-D wave and whole system refurbishes itself (see Usher \& Craik 1975). Note that although the background disturbance level may be high in the flows considered here, as they are turbulent, the organised disturbances are small and hence we would expect C-type and not H-type structures.

For the 2-D problem, since the organised disturbance is solenoidal we can define a stream function $\psi$ such that $\tilde{u}=\partial \psi / \partial y$ and $\tilde{v}=-\partial \psi / \partial x$ and $\psi$ may be written as,

$$
\psi=\phi(y) \exp ^{[\iota \alpha(x-c t)]} .
$$

Substituting (11) in (8) and modelling the Reynolds stresses as above we obtain the following extended Orr-Sommerfeld equation as in $\mathrm{S} \& \mathrm{~V}$, 


$$
\begin{gathered}
i \alpha\left[(\bar{u}-c)\left(\phi^{\prime \prime}-\alpha^{2} \phi\right)-\bar{u}^{\prime \prime} \phi\right]-1 / \operatorname{Re}\left[\phi^{\prime \prime \prime \prime}-2 \alpha^{2} \phi^{\prime \prime}+\alpha^{4} \phi\right] \\
-1 / \operatorname{Re}\left[E\left\{\phi^{\prime \prime \prime \prime}-2 \alpha^{2} \phi^{\prime \prime}+\alpha^{4} \phi\right\}+2 E^{\prime}\left\{\phi^{\prime \prime \prime}-\alpha^{2} \phi^{\prime}\right\}\right. \\
\left.+E^{\prime \prime}\left\{\phi^{\prime \prime}+\alpha^{2} \phi\right\}\right]-\lambda E / \operatorname{Re}\left[-2 i \alpha \phi^{\prime \prime \prime}+2 i \alpha^{3} \phi^{\prime}\right] \\
-2 i \alpha \phi^{\prime} / \operatorname{Re}\left[\lambda E^{\prime \prime}+2 \lambda^{\prime} E^{\prime}+\lambda^{\prime \prime} E\right]=0 .
\end{gathered}
$$

Primes $\left({ }^{\prime}\right)$ denote differentiation with respect to $y$. (Note that the same equation as (12) above, reported in $\mathrm{S} \& \mathrm{~V}$ has the wrong sign for the two terms involving $\lambda$ and its derivatives. However, the computations were done with the proper sign and hence the numerical results reported in $\mathrm{S} \& \mathrm{~V}$ remain unchanged. The same error was carried over in $\mathrm{S} \& \mathrm{~V}$ to the equation scaled in inner variables.) We note that the eddy viscosity used in (6) and (7) has been non-dimensionalised by the mean velocity, $V$, and channel half-width, $H$. However, in (12) the eddy viscosity has been non-dimensionalised by the molecular viscosity, $\nu$, which is more customary and this is denoted by $E$.

In (12), the first group of terms in square brackets corresponds to the Rayleigh equation; the first two groups of terms in square brackets correspond to the classical Orr-Sommerfeld equation; and, the remaining terms constitute the modifications in the classical OrrSommerfeld equation.

The reader is referred to S \& V and Sen \& Veeravalli (1994) for a more detailed derivation and for a discussion of the subtleties involved in the model.

The boundary conditions at the wall $y=0$ are,

$$
\phi=0 ; \phi^{\prime}=0 \text {. }
$$

And at the channel centre $y=1$ are,

$$
\begin{aligned}
\phi^{\prime} & =0 ; & \phi^{\prime \prime \prime}=0, & \text { anti-symmetric mode; } \\
\phi & =0 ; & \phi^{\prime \prime}=0, & \text { symmetric mode. }
\end{aligned}
$$

Equation (12) may also be written in inner variables using the friction velocity $v_{*}$ and the length scale $\nu / v_{*}$. We denote quantities that are non-dimensionalised by the inner scales with the superscript $(+)$. Then, $\lambda=\lambda^{+} ; E=E^{+} ; B \equiv v_{*}^{2} / V^{2} ; y^{+}=y \operatorname{Re} \sqrt{B} ; \bar{u}^{+}=\bar{u} / v_{*}$; and, $\alpha^{+}=\alpha /(\operatorname{Re} \sqrt{B})$, where $B$ is the non-dimensional wall shear stress. Thus (12) becomes

$$
\begin{aligned}
& i \alpha^{+}\left[\left(\bar{u}^{+}-c^{+}\right)\left(\phi^{\prime \prime}-\alpha^{+^{2}} \phi\right)-\bar{u}^{+\prime \prime} \phi\right]-\left[\phi^{\prime \prime \prime \prime}-2 \alpha^{+^{2}} \phi^{\prime \prime}+\alpha^{+^{4}} \phi\right] \\
& -\left[E^{+}\left\{\phi^{\prime \prime \prime \prime}-2 \alpha^{+^{2}} \phi^{\prime \prime}+\alpha^{+^{4}} \phi\right\}+2 E^{+^{\prime}}\left\{\phi^{\prime \prime \prime}-\alpha^{+^{2}} \phi^{\prime}\right\}\right. \\
& \left.+E^{+\prime \prime}\left\{\phi^{\prime \prime}+\alpha^{+2} \phi\right\}\right]-\lambda^{+} E^{+}\left[-2 i \alpha^{+} \phi^{\prime \prime \prime}+2 i \alpha^{+3} \phi^{\prime}\right] \\
& -2 i \alpha^{+} \phi^{\prime}\left[\lambda^{+} E^{+\prime \prime}+2 \lambda^{+^{\prime}} E^{+^{\prime}}+{\lambda^{+}}^{\prime \prime} E^{+}\right]=0 \text {. }
\end{aligned}
$$

Here primes denote differentiation with respect to $y^{+}$. In (14) there is no explicit dependence on Reynolds number. However, Reynolds number does play a role due to the behaviour of $E^{+}$and $\lambda^{+}$in the outer region. Finally, if the unstable modes are wall modes, then (14) shows that their behaviour should be universal at high Reynolds numbers and this is confirmed by the calculations as shown below.

In order to compute the eigenfunction, expressions for the eddy viscosity, $E$, and the anisotropy function, $\lambda$, as functions of $y$ are required. Reynolds \& Tiederman (1967) report an analytical expression for $E$ which matches well with experimental measurements. 
The same expression is used here and is as follows:

$$
\begin{gathered}
E(y)=\frac{1}{2}\left[1+\kappa^{2} \operatorname{Re}^{2} B / 9\left(2 y-y^{2}\right)^{2}\left(3-4 y+2 y^{2}\right)^{2}\right. \\
\left.\times\left\{1-\exp \left(-y \operatorname{Re} \sqrt{B} / A^{+}\right)\right\}^{2}\right]^{\frac{1}{2}}-\frac{1}{2}
\end{gathered}
$$

In equation (15) $\kappa$ is the von Kármán constant and $A^{+}$is the Van Driest constant. Here the values used for $\kappa$ and $A^{+}$are 0.4 and 27 respectively.

In $\mathrm{S} \& \mathrm{~V}$ two expressions were given for $\lambda$ following a detailed discussion on how it is obtained from experimental data (Klebenoff 1954) and from numerical simulations (Spalart 1988). The first is based on inner variables and is as follows:

$$
\begin{aligned}
\lambda\left(y^{+}\right)= & \lambda_{h i}\left[\lambda_{l o} / \lambda_{h i}+\left(\frac{1-\lambda_{l o} / \lambda_{h i}}{2}\right)\left\{1-\operatorname{erf}\left(\frac{y^{+}-y_{0}^{+}}{\sigma}\right)\right\}\right], \\
& \text { with } \lambda_{h i}=10.5, \lambda_{l o}=3.0, y_{0}^{+}=18.0, \text { and } \sigma=7.5 .
\end{aligned}
$$

The second expression is smoother and is easier to use in computations and is given in outer variables as:

$$
\begin{aligned}
\lambda(y)= & \lambda_{h i}\left[\lambda_{l o} / \lambda_{h i}+\left(\frac{1-\lambda_{l o} / \lambda_{h i}}{2}\right)\left\{1-\operatorname{erf}\left(\frac{y-y_{0}}{\sigma}\right)\right\}\right], \\
& \text { with } \lambda_{h i}=10.0, \lambda_{l o}=3.0, y_{0}=0.5, \text { and } \sigma=0.25 .
\end{aligned}
$$

Expression (17) is easier to use from the point of view of computation, however, it does not match well with experimental data. Hence, for comparison with the experimental results of Hussain \& Reynolds (1972), on the behaviour of an organised wave in turbulent channel flow, it is (16) that is more relevant. We note further, that both (16) and (17) yield a value of 3 for $\lambda$ at the centre of the channel. However, at the centre of the channel the turbulence is nearly isotropic and hence $\lambda$ should tend to zero there, as is confirmed by experimental data. This error in (16) and (17) is not relevant if one is interested in wall modes. However, for the calculation of outer modes, clearly (16) and (17) are not correct. Thus, for comparison with the experiments of Hussain \& Reynolds (1972), (16) has been modified as:

$$
\lambda=f\left(y^{+}\right) g(y)
$$

where

$$
f\left(y^{+}\right)=10.5\left[3 / 10.5+\left(\frac{1-3 / 10.5}{2}\right)\left\{1-\operatorname{erf}\left(\frac{y^{+}-18.0}{7.5}\right)\right\}\right]
$$

and

$$
g(y)=0.5[1-\operatorname{erf}(y-0.25 / 0.075)]
$$

Note that for a proper match with experiments it is essential that the approach of $\lambda$ to zero be expressed in outer variables and thus the need for a composite expression.

Figure 3 shows the resulting variation of $\lambda$ with $y$ at a Reynolds number of 5000. Also shown in figure 3 is $\lambda$ estimated from the experimental data of Klebanoff (1954). The rather high value of $\lambda \approx 25$ shown by the experimental data near the wall is not critical to the model and we find that good results are obtained by allowing $\lambda$ to start from a value of 10.5 at the wall. 


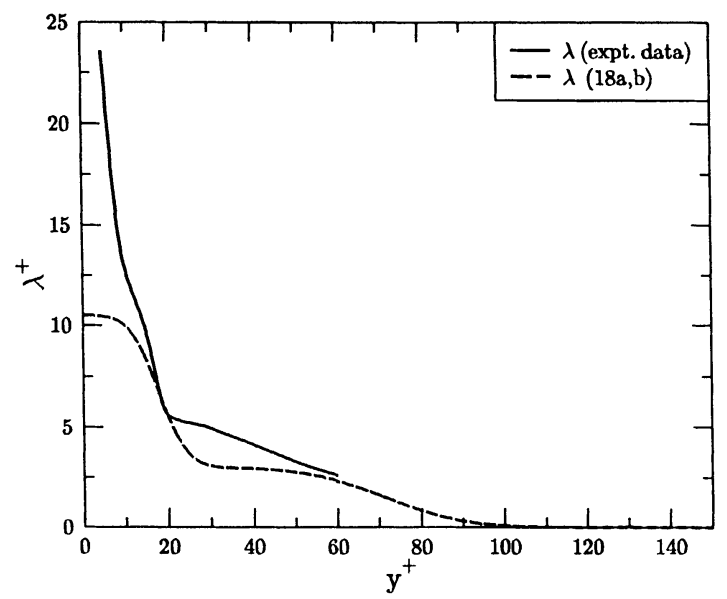

Figure 3. Graph of anisotropy function $\lambda^{+}(=\lambda)$ versus $y^{+}$, standardised in terms of inner variables. Based on experimental data of Klebanoff (1954), and from $(18 a, b)$.

Finally, an analytically continuous and realistic turbulent mean velocity profile is required. This is obtained by integrating the mean momentum equation,

$$
\frac{\mathrm{d}}{\mathrm{d} y}\left((1+E) \frac{\mathrm{d} \bar{u}}{\mathrm{~d} y}\right)=-\operatorname{Re} B
$$

Thus,

$$
(1+E) \frac{\mathrm{d} \bar{u}}{\mathrm{~d} y}=\operatorname{Re} B(1-y) .
$$

Given the Reynolds number, a starting value for $B$ is chosen and (20) is integrated to get $\bar{u}(y)$. If the average velocity, $V$, is not equal to 1 , then $B$ is suitably modified and the procedure is iterated till the condition $V=1$ is satisfied. This is the same as the procedure outlined in Reynolds \& Tiederman (1967).

\section{Results and discussion}

Details of the numerical scheme used to compute the eigenvalues are given in Sen \& Vashist (1989) and Sen (1993). The calculations were made only for symmetric modes as they are more unstable. A value for $\alpha$ was chosen and the complex wave speed, $c$, was computed as the eigenvalue. A positive value for $c_{i}$, thus, indicates instability.

\subsection{Comparison with boundary layer calculations}

Calculations were done at three different Reynolds numbers - 5000, 11000 and 17000 for comparison with the boundary layer calculations of $\mathrm{S} \& \mathrm{~V}$. The results presented in this section, unless specified otherwise, have been computed with $\lambda$ as in (17) - the expression in outer variables wherein $\lambda$ varies more gently with $y$. 


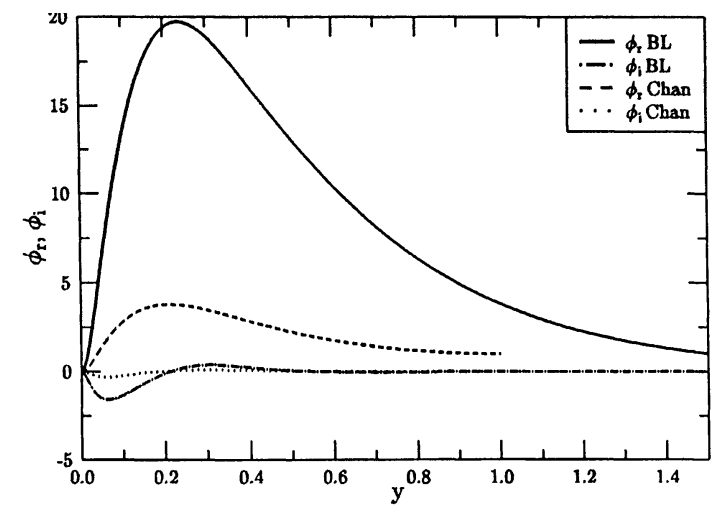

Figure 4. Graph of eigenfunction $\phi=\phi_{r}+i \phi_{i}$ versus y. Normalisation $\phi=1+0_{i}$ at $y=1.0$ for channel flow and $\operatorname{Re}=5000, \alpha=3.15, c_{r}=0.3816348, c_{i}=0.0040752$. Normalisation $\phi=1+0_{i}$ at $y=1.5$ for the boundary layer and $\operatorname{Re}=5000, \alpha=2.7, c_{r}=0.3486034, c_{i}=0.0044436$. (Expression (17) has been used for $\lambda$.)

In each case a wide range of unstable wave numbers was obtained. The value of $c_{r}$ was small (less than 0.4) indicating that the unstable modes are wall modes. Figure 4 shows a typical eigenfunction obtained for $\operatorname{Re}=5000$ and $\alpha=3.15$, with $c_{r}=0.381634$ and $c_{i}=0.0040752$. The eigenfunction has been normalised such that $\phi=1+0 \iota$ at $y=1$. The corresponding curves for the boundary layer, at the same Reynolds number and nearly the same wavenumber, are also shown in the same figure. Curves of the two sets are qualitatively very similar to each other. The difference in magnitude does not matter as the vertical scale is arbitrary. Figure 5 shows the behaviour of the root mean square (r.m.s.) values of $u$ r.m.s. and $v$ r.m.s. respectively, at the same parameter values as above, for both

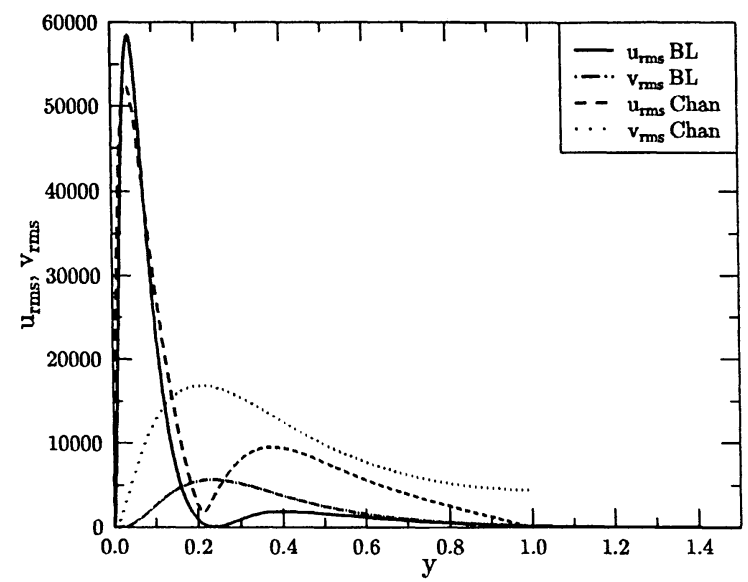

Figure 5. Graph of root mean square values of $u$ and $v$ versus $y$. Normalisation, $\phi=1+0_{i}$ at $y=1.0$ for channel flow and $\operatorname{Re}=5000, \alpha=3.15, c_{r}=0.3816348, c_{i}=0.0040752$. Normalisation $\phi=1+0_{i}$ at $y=1.5$ for the boundary layer and $\operatorname{Re}=5000, \alpha=2.7, \quad c_{r}=3486034$, $c_{i}=0.0044436$. (The channel flow curves have been scaled up by 1000 for comparison.) [Expression (17) has been used for $\lambda$.] 


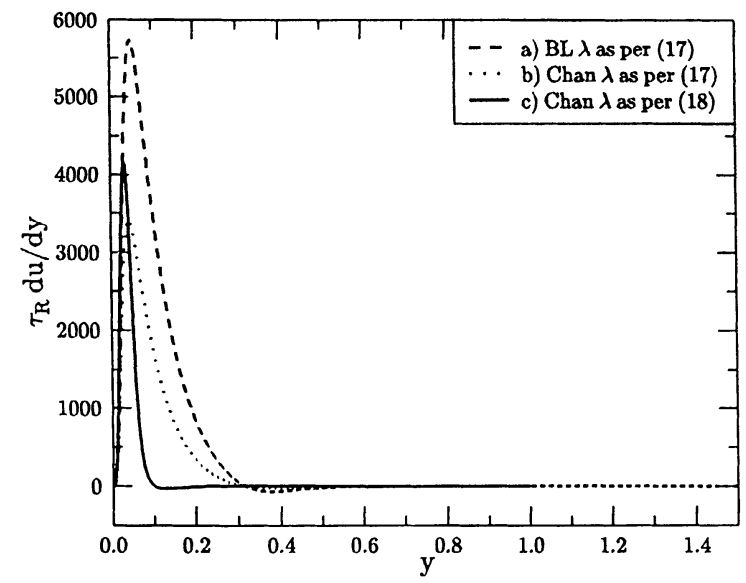

Figure 6. Graph of $\tau_{R} \mathrm{~d} \bar{u} / \mathrm{d} y$ (production) versus $y$. a) Normalisation $\phi=1+0_{i}$ at $y=1.5$ for the boundary layer and $\operatorname{Re}=5000, \alpha=2.7, c_{r}=0.3486034, c_{i}=0.0044436$. b) Normalisation, $\phi=1+0_{i}$ at $y=1.0$ for channel flow and $\operatorname{Re}=5000, \alpha=3.15, c_{r}=0.3816348, c_{i}=0.0040752$. c) Channel flow; $\lambda$ calculated using expression (18) and $\operatorname{Re}=5000, \alpha=9.434, c_{r}=0.28762$, $c_{i}=0.001693$ (vertical scale is arbitrary).

the channel flow and the boundary layer. Again since the dominant modes are wall modes, the qualitative nature of the two sets of curves is the same. However, we note that the relative magnitude of $v$ r.m.s. is larger for the channel flow. The $u$ r.m.s. curves peak at $y^{+} \approx 11$ and $y^{+} \approx 10.5$ for the boundary layer and channel flow respectively, and this agrees very well with experimental data (see Tennekes \& Lumley 1972) and numerical simulations (Spalart 1988). The results obtained with $\lambda$ given by (18) are qualitatively the same but the $c_{r}$ values are slightly smaller and the peak of $u$ r.m.s. occurs at a lower value of $y^{+}$.

Figure 6 shows the profile of the production term, $\tau_{R} \bar{u}^{\prime}$, where, $\tau_{R}=\overline{\tilde{u}} \tilde{v}$. Again the curves for both the channel flow and the boundary layer are qualitatively the same. The peak lies at $y^{+} \approx 12.5$ for the boundary layer and at $y^{+} \approx 12.25$ for the channel flow. Again this agrees very well with experimental data. Also shown in this figure is a typical result using (18) for $\lambda$. This is in qualitative agreement with the other two curves but, as is to be expected, the peak lies closer to the wall.

Figure 7 shows the variation of the growth rate of the organised disturbance, $\alpha^{+} c_{i}^{+}$, with the wavenumber $\alpha^{+}$. Since the unstable modes are wall modes we find that the curves are nearly the same at all the three Reynolds numbers considered here. The separation between the curves for $\mathrm{Re}=5000$ and $\mathrm{Re}=11000$ is much greater than that between the curves for $\operatorname{Re}=11000$ and $\operatorname{Re}=17000$, indicating a rapid approach to a limiting curve. Further, the curves for the two different flows fall literally on top of each other. This is consistent with (14) above.

Figure 8 shows a comparison of the growth rate curves, similar to those shown in figure 7 , but here, $\lambda$ given by (16) has been used in the boundary-layer calculation and $\lambda$ given by (18) has been used in the channel flow calculation. In either case the Reynolds number is 5000. The band of unstable wave numbers is smaller than that seen in figure 7, but again the curves for the two flows are nearly indistinguishable.

The conclusion that may be drawn from figures 7 and 8 is that, since the unstable modes are wall modes, neither the outer boundary condition on the velocity field nor the behaviour of $\lambda$ in the outer region, has any significant bearing on the stability characteristics. 


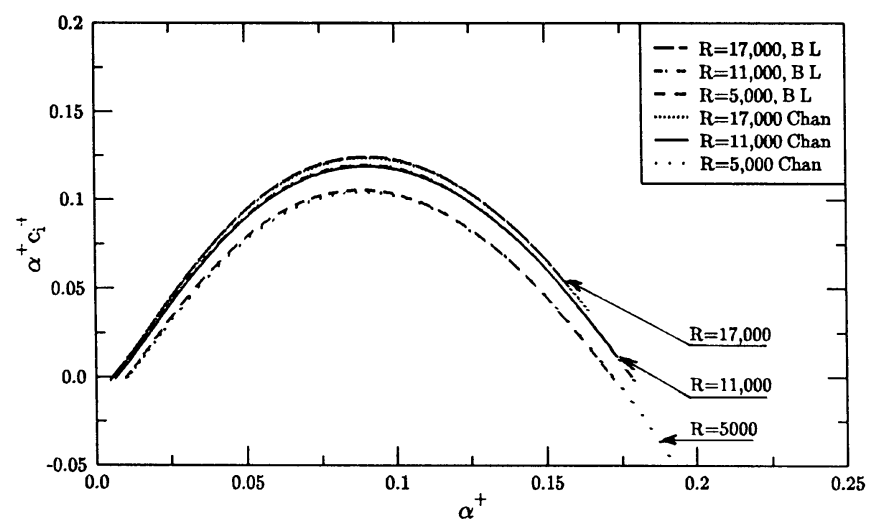

Figure 7. Graph of the growth rate, $\alpha^{+} c_{i}^{+}$versus $\alpha^{+}$in inner variables scaling, at different Reynolds numbers.

\subsection{Comparison with experiments}

As far as we are aware, the only experimental results with which direct comparisons may be made are those of Hussain \& Reynolds (1972) (see also Hussain \& Reynolds 1970) in a turbulent channel. Hussain \& Reynolds (1972) introduced a 2-D disturbance in a fully developed turbulent channel using a vibrating ribbon at four different frequencies and extracted the resulting eigenfunctions and their amplitudes and phases using phase-locked averaging. However, all the disturbances considered there (the minimum frequency chosen was $25 \mathrm{~Hz}$ and the maximum frequency was $100 \mathrm{~Hz}$ ) were found to be damped outer (since $c_{r}$ was found to be close to 1.0) modes. The average velocity, $V$, used in the experiment was $5.88 \mathrm{~m} / \mathrm{s}$ and the channel half-width, $\delta$ was approximately $3.2 \mathrm{~cm}$ yielding a Reynolds number of 12160 .

Given the parameters as in Hussain \& Reynolds (1972), we can easily estimate from figure 7 (where (17) has been used for $\lambda$ ) that a band of unstable waves (inner mode) exists

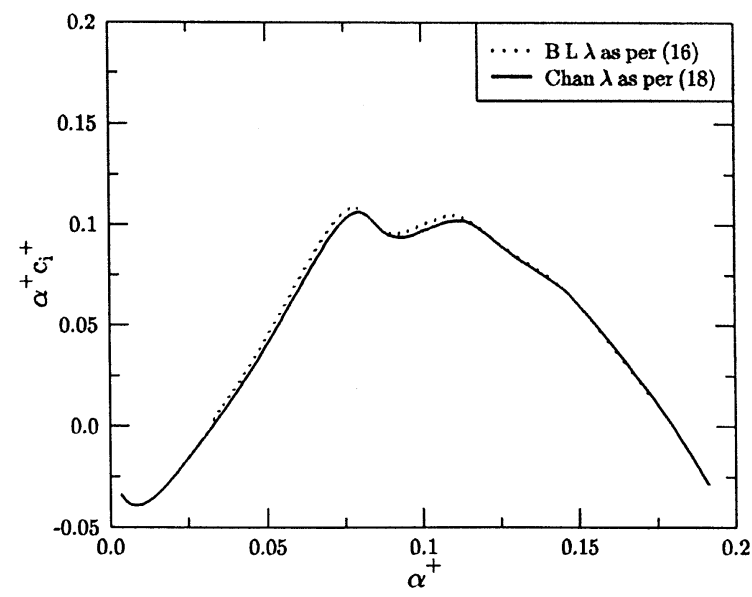

Figure 8. Graph of the growth rate, $\alpha^{+} c_{i}^{+}$versus $\alpha^{+}$(inner variables scaling). The Reynolds number is 5000 . 
for a frequency range from $46 \mathrm{~Hz}$ to $1400 \mathrm{~Hz}$, with the peak at approximately $770 \mathrm{~Hz}$. However, from figure 8 , (where (18) has been used for $\lambda$ ), the frequency range for unstable waves is $150 \mathrm{~Hz}$ to $1000 \mathrm{~Hz}$, with the primary peak at approximately $440 \mathrm{~Hz}$ and this is in agreement with the experimental observation that even at a frequency of $100 \mathrm{~Hz}$ the disturbance was damped. We note that while (17) is more convenient for computations, it is (18) that is closer to numerical and laboratory experiments. Thus for comparison with the experimental data of Hussain \& Reynolds (1972) it is (18) that is to be used. Our computations then show that in essence there exist two modes; an inner mode (with $c_{r}$ close to 0.3 ) whose behaviour is as shown in figure 8 and an outer mode (with $c_{r}$ close to 1.0) which is always damped (there exist additional outer modes which are more heavily damped which will not be of concern here). The results are summarised in table 1.

The temporal growth rate exponent in the computations is estimated as $\alpha c_{i}$ since a real $\alpha$ is chosen. However, in the experiment it is the frequency $\alpha c$ that is real, and $\alpha$ is a complex number. Thus the growth rate exponent in the experiment is $-\alpha_{i} x$. We can convert this to a temporal growth rate by writing $x=V_{g} t$, where, $V_{g}$ is the group velocity, given by $V_{g}=\mathrm{d}(c \alpha) / \mathrm{d}(\alpha)$. However, since for both the inner and outer modes we find that $c_{r}$ varies very slowly with $\alpha$, the group velocity $V_{g}$ is approximately equal to $c_{r}$, from which we can estimate the equivalent temporal growth rate exponent in the experiment as $-\alpha_{i} c_{r}$. This is the growth rate reported in table 1 .

We note from table 1 that there is reasonable qualitative agreement between the experimental results of Hussain \& Reynolds (1972) and our computational results for the outer mode. Secondly, for the range of frequencies studied, the inner mode is more heavily damped and thus only the outer mode is picked up by the experiments of Hussain \& Reynolds (1972).

While there is agreement between the experiment and our computation for the parameters shown in table 1 , other features do not match so readily. The profile for $u$ rms determined in Hussain \& Reynolds (1972) looks qualitatively similar to the one shown in figure 5, close to the vibrating ribbon, and the hump close to the wall gradually disappears as $x$ increases. Figure 9 shows the profile for $u$ r.m.s. obtained for the outer mode corresponding to a frequency of $50.7 \mathrm{~Hz}$. We note that this is qualitatively dissimilar to the

Table 1. Wave data: Comparison between experiments of Hussain \& Reynolds (1972) (H\&R) and present calculations.

All quantities except the frequency are dimensionless and normalised using the half width and the mean velocity.

\begin{tabular}{lcccc}
\hline Frequency $(\mathrm{Hz})$ & $\alpha_{r}$ & $c_{r}$ & Growth rate & Source \\
\hline 25 & $0.981 \pm 13 \%$ & $0.861 \pm 13 \%$ & $-0.063 \pm 23 \%$ & H\&R \\
50 & $1.87 \pm 8.5 \%$ & $0.904 \pm 8.5 \%$ & $-0.11 \pm 20.5 \%$ & H\&R \\
75 & $2.72 \pm 7 \%$ & $0.93 \pm 7 \%$ & $-0.18 \pm 21 \%$ & H\&R \\
100 & $3.57 \pm 6.1 \%$ & $0.946 \pm 6.1 \%$ & $-0.27 \pm 22 \%$ & H\&R \\
24.5 & 0.85 & 0.98 & -0.049 & Outer mode \\
50.7 & 1.68 & 1.02 & -0.041 & Outer mode \\
77.0 & 2.49 & 1.05 & -0.052 & Outer mode \\
99.6 & 3.18 & 1.06 & -0.085 & Outer mode \\
25.5 & 1.69 & 0.265 & -0.62 & Inner mode \\
49.6 & 3.60 & 0.242 & -0.60 & Inner mode \\
74.3 & 5.11 & 0.255 & -0.47 & Inner mode \\
98.1 & 6.45 & 0.267 & -0.33 & Inner mode \\
\hline
\end{tabular}




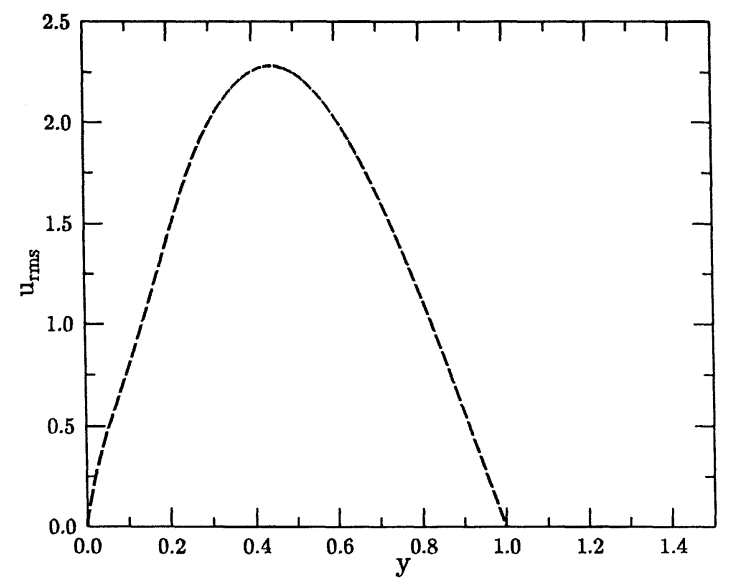

Figure 9. Graph of r.m.s. value of $u$ versus $y$. Normalisation, $\phi=1+0_{i}$ at $y=1.0 . \operatorname{Re}=12160$, $\alpha=1.675, c_{r}=1.02087, c_{i}=-0.02426$. (Expression (18) has been used for $\lambda$.)

profile determined in the experiment since it has only a single maximum. The same disagreement was noted by Reynolds \& Hussain (1972). Reynolds \& Hussain (1972) tried to resolve this by noting that there is a second outer mode that is more heavily damped and if initially the ribbon excites both the modes then one could obtain a double humped profile as seen in the experiments with the hump near the wall getting suppressed as $x$ increases, because it is more heavily damped. However, as shown in Reynolds \& Hussain (1972) even with the superposition of the outer modes, the locations of the two maxima do not agree with the experimentally observed values. It is likely that it is a superposition of the outer and inner modes determined here that is more appropriate, especially at $100 \mathrm{~Hz}$, since the inner mode is only slightly more damped than the outer mode at this frequency. This can be properly resolved only after conducting further experiments at higher frequencies designed to match those of the unstable inner mode.

Finally, we note from the spectral measurements of Hussain \& Reynolds (1975), that the band of unstable wavenumbers obtained in figures 7 and 8, is fully contained in the energycontaining eddies region (figure not shown). The same result was reported in $\mathrm{S} \& \mathrm{~V}$ for the turbulent boundary layer.

\section{Conclusions}

We have shown that the extended Orr-Sommerfeld equation developed in S \& V, captures some of the key features of both turbulent channel flow and the turbulent boundary-layer. For example, the location of the peak in the $u$ r.m.s. and production curves matches well with experimental data. Further, the growth rate curves for the two flows are virtually identical, when plotted in inner variables, which is what one would expect from the physics of wall-bounded shear flows at high Reynolds numbers. The growth rate curves are also insensitive to the behaviour of $\lambda$ in the outer region. This lends credence to the model used in obtaining the extended Orr-Sommerfeld equation. In brief, we have a viable mechanism to explain the sustenance of turbulence in fully-developed wall-bounded turbulent flows. 
Comparisons with the experiments of Hussain \& Reynolds (1972) indicate a reasonable qualitative agreement. Our computations show that we would have to use higher frequencies than those used in Hussain \& Reynolds (1972) in order to capture the unstable inner mode. Further experimentation is required to fully resolve this, which is currently underway.

This project was funded by the Council of Scientific and Industrial Research, New Delhi. We are grateful to Dr S Mallick, Human Resource Development Group, CSIR for his support. We are also grateful to Professor R Narasimha, FRS for his constant encouragement and guidance.

\section{Appendix A: Squire's theorem}

Consider 3-D normal modes of the form

$$
\tilde{u}_{i}=\hat{u}_{i}(y) \exp ^{[\iota(\alpha x+\beta z-c \alpha t)]} .
$$

Then the momentum equation (8) yields

$$
\begin{aligned}
& \iota \alpha(\bar{u}-c) \hat{u}+\frac{(1+E)}{\operatorname{Re}}\left(\alpha^{2}+\beta^{2}\right) \hat{u}+\bar{u}^{\prime} \hat{v}=-\iota \alpha \hat{p}+\frac{E^{\prime}}{\operatorname{Re}}\left(\hat{u}^{\prime}+\iota \alpha \hat{v}\right) \\
& \quad-\frac{(E \lambda)^{\prime}}{\operatorname{Re}}\left(\hat{v}^{\prime}-\iota \alpha \hat{u}\right)+\frac{(1+E)}{\operatorname{Re}} \hat{u}^{\prime \prime}, \\
& \iota \alpha(\bar{u}-c) \hat{v}+\frac{(1+E)}{\operatorname{Re}}\left(\alpha^{2}+\beta^{2}\right) \hat{v}=-\hat{p}^{\prime}+\frac{2 E^{\prime}}{\operatorname{Re}} \hat{v}^{\prime}+\frac{2(E \lambda)^{\prime}}{\operatorname{Re}} \hat{u}^{\prime}+\frac{(1+E)}{\operatorname{Re}} \hat{v}^{\prime \prime} \\
& \quad+\frac{E \lambda}{\operatorname{Re}}\left(-\left(\alpha^{2}+\beta^{2}\right) \hat{u}+2 \hat{u}^{\prime \prime}-\iota \alpha \hat{v}^{\prime}\right), \\
& \iota \alpha(\bar{u}-c) \hat{w}+\frac{(1+E)}{\operatorname{Re}}\left(\alpha^{2}+\beta^{2}\right) \hat{w}=-\iota \beta \hat{p}+\frac{E^{\prime}}{\operatorname{Re}}\left(\hat{w}^{\prime}+\iota \beta \hat{v}\right)+\frac{(E \lambda)^{\prime}}{\operatorname{Re}} \iota \beta \hat{u} \\
& \quad+\frac{(1+E)}{\operatorname{Re}} \hat{w}^{\prime \prime}+\frac{E \lambda}{\operatorname{Re}}\left(\iota \beta \hat{u}^{\prime}-\iota \alpha \hat{w}^{\prime}\right) .
\end{aligned}
$$

The continuity equation becomes,

$$
\iota \alpha \hat{u}+\hat{v}^{\prime}+\iota \beta \hat{w}=0 .
$$

If we now consider only $2-\mathrm{D}$ disturbances (i.e. $\beta=0$ and $\hat{w}=0)$ then $(\mathrm{A} 2 \mathrm{a})$ and $(\mathrm{A} 2 \mathrm{~b})$ become,

$$
\begin{aligned}
& \iota \alpha(\bar{u}-c) \hat{u}+\frac{(1+E)}{\operatorname{Re}} \alpha^{2} \hat{u}+\bar{u}^{\prime} \hat{v}=-\iota \alpha \hat{p}+\frac{E^{\prime}}{\operatorname{Re}}\left(\hat{u}^{\prime}+\iota \alpha \hat{v}\right) \\
& -\frac{(E \lambda)^{\prime}}{\operatorname{Re}}\left(\hat{v}^{\prime}-\iota \alpha \hat{u}\right)+\frac{(1+E)}{\operatorname{Re}} \hat{u}^{\prime \prime},
\end{aligned}
$$

and

$$
\begin{aligned}
& \iota \alpha(\bar{u}-c) \hat{v}+\frac{(1+E)}{\operatorname{Re}} \alpha^{2} \hat{v}=-\hat{p}^{\prime}+\frac{2 E^{\prime}}{\operatorname{Re}} \hat{v}^{\prime}+\frac{2(E \lambda)^{\prime}}{\operatorname{Re}} \hat{u}^{\prime}+\frac{(1+E)}{\operatorname{Re}} \hat{v}^{\prime \prime} \\
& \quad+\frac{E \lambda}{\operatorname{Re}}\left(-\alpha^{2} \hat{u}+2 \hat{u}^{\prime \prime}-\iota \alpha \hat{v}^{\prime}\right) .
\end{aligned}
$$


The continuity equation becomes,

$$
\iota \alpha \hat{u}+\hat{v}^{\prime}=0
$$

Using Squire's transformation given by,

$$
\alpha^{*}=\left(\alpha^{2}+\beta^{2}\right)^{1 / 2} ; \quad \alpha^{*} u^{*}=\alpha \hat{u}+\beta \hat{w} ; \quad \alpha^{*} \operatorname{Re}^{*}=\alpha \operatorname{Re} \text { etc },
$$

(A2) may be written as,

$$
\begin{aligned}
& \iota \alpha^{*}(\bar{u}-c) u^{*}+\frac{(1+E)}{\operatorname{Re}^{*}} \alpha^{* 2} \hat{u}+\bar{u}^{\prime} v^{*}=-\iota \alpha^{*} p^{*}+\frac{E^{\prime}}{\operatorname{Re}^{*}}\left(u^{* \prime}+\iota \alpha^{*} v^{*}\right) \\
& -\frac{(E \lambda)^{\prime}}{\operatorname{Re}^{*}}\left(v^{* \prime}-\iota \alpha^{*} u^{*}\right)+\frac{(1+E)}{\operatorname{Re}^{*}} u^{* \prime \prime} \\
& +\left[\frac{(E \lambda)^{\prime}}{\operatorname{Re}^{*}}\left(-v^{*}\left(\frac{\alpha}{\alpha^{*}}-1\right)+\iota \alpha^{*}\left(\hat{u}-u^{*}\right)\right)+\frac{E \lambda}{\operatorname{Re}^{*}} \frac{\iota \beta}{\alpha^{*}}\left(\beta \hat{u}^{\prime}-\alpha \hat{w}^{\prime}\right)\right], \quad(\mathrm{A} 4 \mathrm{a}) \\
& \iota \alpha^{*}(\bar{u}-c) v^{*}+\frac{(1+E)}{\operatorname{Re}^{*}} \alpha^{* 2} v^{*}=-p^{* \prime}+\frac{2 E^{\prime}}{\operatorname{Re}^{*}} v^{* \prime}+\frac{2(E \lambda)^{\prime}}{\operatorname{Re}^{*}} u^{* \prime}+\frac{(1+E)}{\operatorname{Re}^{*}} v^{* \prime \prime} \\
& +\frac{E \lambda}{\operatorname{Re}^{*}}\left(-\alpha^{* 2} u^{*}+2 u^{* \prime \prime}-\iota \alpha^{*} v^{* \prime}\right) \\
& +\left[\frac{E \lambda}{\operatorname{Re}^{*}}\left(-\alpha^{* 2}\left(\hat{u}-u^{*}\right)+2\left(\hat{u}^{\prime \prime}-u^{* \prime \prime}\right)-\iota\left(\alpha-\alpha^{*}\right) v^{* \prime}\right)+2 \frac{(E \lambda)^{\prime}}{\operatorname{Re}^{*}}\left(\hat{u}^{\prime}-u^{* \prime}\right)\right],
\end{aligned}
$$

and

$$
\iota \alpha^{*} u^{*}+v^{* \prime}=0
$$

Comparing (A3) and (A4) we find that the terms in the square brackets in (A4) are the problematic ones. If we now restrict ourselves to near 2-D modes i.e. we restrict $\alpha^{*}$ to a small angle $\theta$ with respect to $\alpha$ as shown in figure 2, and we have the following:

$$
\begin{aligned}
& \alpha=\alpha^{*} \cos \theta \approx \alpha^{*} ; \quad \beta=\alpha^{*} \sin \theta \approx \alpha^{*} \theta \\
& u^{*}=\hat{u} \cos \theta+\hat{w} \sin \theta \approx \hat{u}+\theta \hat{w} \text { etc. }
\end{aligned}
$$

Using these approximations, the term in square brackets in (A4a) may be written as,

$$
\frac{(E \lambda)^{\prime}}{\operatorname{Re}^{*}}\left(v^{*}\left(\frac{\theta^{2}}{2}\right)-\iota \alpha^{*}(\theta \hat{w})\right)+\frac{E \lambda}{\operatorname{Re}^{*}}\left(\iota \theta\left(\theta \alpha^{*} \hat{u}^{\prime}-\alpha^{*} \hat{w}^{\prime}\right)\right) .
$$

Thus we see that the problematic term in (A4a) is at best of order $\theta$. Further, by casting the problem in the direction of the oblique wave (i.e. making an angle $\theta$ with the $x$ axis) and perpendicular to it, it may be shown that $\hat{w}$ itself is of order $\theta$ compared to $\hat{u}$ and $\hat{v}$. Thus the more correct order of magnitude of the term shown in (A6) is $\theta^{2}$. The same conclusion may be drawn with the problematic term in (A4b) also.

Thus we can conclude that if $\theta$ is small, the modified Orr-Sommerfeld equation derived here obeys Squire's theorem and that among near 2-D modes it is the exactly 2-D mode that is the most unstable. 


\section{References}

Craik A D D 1971 Non-linear resonant instability in boundary layers. J. Fluid Mech. 50: 393-413

Gaster M, Kit E, Wygnanski I 1985 Large-scale structures in a forced turbulent mixing layer. J. Fluid Mech. 150: 23-39

Hussain A K M F, Reynolds W C 1970 The mechanics of an organised wave in turbulent shear flow. J. Fluid Mech. 41: 241-258

Hussain A K M F, Reynolds W C 1972 The mechanics of an organised wave in turbulent shear flow. Part 2. Experimental results. J. Fluid Mech. 54: 241-261

Hussain A K M F, Reynolds W C 1975 Measurements in fully developed turbulent channel flow. J. Fluids Eng. 97: 568-578

Klebanoff P S 1954 Characteristics of turbulence in a boundary-layer with zero pressure gradient. NACA technical report No. 3178

Liu J T C 1988 Contributions to the understanding of large-scale coherent structures in developing free turbulent shear flows. Adv. Appl. Mech. 26: 183-309

Malkus W V R 1956 Outline of a theory of turbulent shear flow. J. Fluid Mech. 1: 521-539

Pope S B 1975 A more general effective viscosity hypothesis. J. Fluid Mech. 72: 331-340

Reynolds W C, Hussain A K M F 1972 The mechanics of an organized disturbance in turbulent shear flow. Part 3. Theoretical models and comparisons with experiments. J. Fluid Mech. 54: 263-288

Reynolds W C, Tiederman W G 1967 Stability of turbulent channel flow, with application to Malkus's theory. J. Fluid Mech. 27: 253-272

Roshko A 1992 Instability and turbulence in shear flows. Theoretical and applied mechanics (eds) S R Bodner, J Singer, A Solan, Z Hussain (New York: Elsevier Science)

Sen P K 1993 Some recent developments in the theory of boundary layer instability. Sädhanā 18: 387-403

Sen P K, Vashist T K 1989 On the nonlinear stability of boundary-layer flow over a flat plate. Proc. R. Soc. London A424: 81-92

Sen P K, Veeravalli S V 1994 Hydrodynamic stability theory applied to wall turbulence. Proc. 21st National Conference on Fluid Mechanics and Fluid Power, Osmania University, Hyderabad, pp TF-1 to TF-6

Sen P K, Veeravalli S V 1998 On the behaviour of organised disturbances in a turbulent boundary layer. Sadhana 23: 167-193

Spalart P R 1988 Direct numerical simulation of a turbulent boundary layer up to $R_{\theta}=1410$. $J$. Fluid Mech. 187: 61-98

Tennekes H, Lumley J L 1972 A first course in turbulence (Cambridge, MA: MIT Press)

Usher J R, Craik A D D 1975 Nonlinear wave interactions in shear flows. Part 2. Third-order theory. J. Fluid. Mech. 70: 437-461 